\title{
THE BERNSTEIN CUBATURE FORMULA REVISED
}

\author{
DAN BĂRBOSU AND GHEORGHE ARDELEAN
}

Received 01 February, 2015

\begin{abstract}
Let $a, b, c, d \in \mathbb{R}$ be given such that $a<b, c<d$. Starting with the Bernstein bivariate approximation formula on $[a, b] \times[c, d]$ a corresponding composite Bernstein type cubature formula is constructed. Its coefficients and an upper bound estimation for the remainder term are established. Numerical examples and comparisons with other known cubature formulas are also provided.
\end{abstract}

2010 Mathematics Subject Classification: 65D32; 41A10

Keywords: Bernstein operator, Bernstein bivariate approximation formula, remainder term

\section{Preliminaries}

Let $\mathbb{N}$ be the set of positive integers and $\mathbb{N}_{0}=\mathbb{N} \cup\{0\}$. It is well known (see for example $[1,2,5,6,8,9])$ that the Bernstein bivariate operators $B_{m, n}: C[0,1] \times[0,1] \rightarrow$ $C[0,1] \times[0,1]$ are defined for any $f \in C[0,1] \times[0,1]$, any $(x, y) \in[0,1] \times[0,1]$ and any $m, n \in \mathbb{N}$ by:

$$
B_{m, n} f(x, y)=\sum_{k=0}^{m} \sum_{j=0}^{n} p_{m, k}(x) p_{n, j}(y) f\left(\frac{k}{m}, \frac{j}{n}\right)
$$

where

$$
p_{m, k}(x)=\left(\begin{array}{c}
m \\
k
\end{array}\right) x^{k}(1-x)^{m-k}, \quad p_{n, j}(x)=\left(\begin{array}{l}
n \\
j
\end{array}\right) y^{j}(1-y)^{n-j}
$$

are the fundamental univariate Bernstein's polynomials.

Note that the bivariate polynomials (1.1) are known as the Bernstein bivariate polynomials of degree $(m, n)$.

For any $f \in C[0,1] \times[0,1],(x, y) \in[0,1] \times[0,1], m, n \in \mathbb{N}$, the equality

$$
f(x, y)=B_{m, n} f(x, y)+R_{m, n} f(x, y)
$$

is known as the Bernstein bivariate approximation formula, $R_{m, n} f$ being its remainder term.

Regarding the remainder term of (1.3) were established the following results. 
Theorem 1 ([5]). For any $f \in C[0,1] \times[0,1]$ the remainder term of (1.3) can be represented under the form:

$$
\begin{aligned}
R_{m, n} f(x, y) & \left.=-\frac{x(1-x)}{m} \sum_{k=0}^{m-1} \sum_{j=0}^{n} p_{m-1, k}(x) p_{n, j}(y)\left[\begin{array}{c}
x, \frac{k}{m}, \frac{k+1}{m} \\
\frac{j}{n}
\end{array}\right] f\right] \\
& \left.-\frac{y(1-y)}{n} \sum_{k=0}^{m} \sum_{j=0}^{n-1} p_{m, k}(x) p_{n-1, j}(y)\left[\begin{array}{c}
\frac{k}{m} \\
y, \frac{j}{k}, \frac{j+1}{n}
\end{array}\right] f\right] \\
& +\frac{x y(1-x)(1-y)}{m n} \sum_{k=0}^{m-1} \sum_{j=0}^{n-1} p_{m-1, k}(x) p_{n-1, j}(y)\left[\begin{array}{c}
x, \frac{k}{m}, \frac{k+1}{m} \\
y, \frac{j}{n}, \frac{j+1}{n}
\end{array} ; f\right],
\end{aligned}
$$

for any $(x, y) \in[0,1] \times[0,1]$, where the brackets denote bivariate divided differences.

Theorem 2 ([5]). Let $p, q \in \mathbb{N}_{0}, a \leq x_{0}<x_{1}<\cdots<x_{p} \leq b, c \leq y_{0}<y_{1}<\cdots<$ $y_{q} \leq d$ and $f:[a, b] \times[c, d]$ be given. Suppose $f \in C^{(p-1, q-1)}[a, b] \times[c, d]$ and there exists $\frac{\partial^{p+q} f}{\partial x^{p} \partial y^{q}}$ on $] a, b[\times] c, d[$. Then, there exists $(\xi, \eta) \in] a, b[\times] c, d[$ such that

$$
\left[\begin{array}{l}
x_{0}, x_{1}, \ldots, x_{p} \\
y_{0}, y_{1}, \ldots, y_{q}
\end{array} ; f\right]=\frac{1}{p ! q !} \frac{\partial^{p+q} f}{\partial x^{p} \partial y^{q}}(\xi, \eta) .
$$

Theorem 3 ([5]). Suppose $f \in C^{2,2}[0,1] \times[0,1]$ and there exists $\frac{\partial^{4} f}{\partial x^{2} \partial y^{2}}$, bounded on $] 0,1[\times] 0,1[$. The following

$$
\left|\left(R_{m, n} f\right)(x, y)\right| \leq \frac{x(1-x)}{2 m} M_{1}[f]+\frac{y(1-y)}{2 n} M_{2}[f]+\frac{x y(1-x)(1-y)}{4 m n} M_{3}[f]
$$

holds, for any $(x, y) \in[0,1] \times[0,1]$ and any $m, n \in \mathbb{N}$, where

$$
\begin{aligned}
& M_{1}(f)=\sup _{(x, y) \in] 0,1[\times] 0,1[}\left|\frac{\partial^{2} f(x, y)}{\partial x^{2}}\right|, \\
& M_{2}(f)=\sup _{(x, y) \in] 0,1[\times] 0,1[}\left|\frac{\partial^{2} f(x, y)}{\partial y^{2}}\right|, \\
& M_{3}(f)=\sup _{(x, y) \in] 0,1[\times] 0,1[}\left|\frac{\partial^{4} f(x, y)}{\partial x^{2} \partial y^{2}}\right| .
\end{aligned}
$$

Applying the above results, in [4] was obtained the following Bernstein type cubature formula. 
Theorem 4 ([3]). Let $f \in C^{2,2}[0,1] \times[0,1]$ be given such that $\frac{\partial^{2} f}{\partial x^{2}}, \frac{\partial^{2} f}{\partial y^{2}}$ and $\frac{\partial^{4} f}{\partial x^{2} \partial y^{2}}$ are bounded on $] 0,1[\times] 0,1[$. Then, the following Bernstein type cubature formula

$$
\int_{0}^{1} \int_{0}^{1} f(x, y) d x d y=\sum_{i=0}^{m} \sum_{j=0}^{n} A_{i, j} f\left(\frac{i}{m}, \frac{j}{n}\right)+R_{m, n}[f]
$$

holds, where the coefficients are expressed by

$$
A_{i j}=\frac{1}{(m+1)(n+1)}, \quad(\forall) i=\overline{0, m},(\forall) j=\overline{0, n}
$$

and the remainder term satisfies

$$
\left|R_{m, n}[f]\right| \leq \frac{1}{12 m} M_{1}[f]+\frac{1}{12 n} M_{2}[f]+\frac{1}{144 m n} M_{3}[f] .
$$

Note that the cubature formula $(1.8)$ has the degree of exactness $(1,1)$ i.e. it is exact for the bivariate polynomials $\epsilon_{i, j}(x, y)=x^{i} y^{j}, 0 \leq i \leq j \leq 1, i, j \in \mathbb{N}_{0}, i+j \leq 1$.

Theorem 1.4 was generalized as follows.

Theorem 5 ([3]). Let $f \in C^{2,2}[0,1] \times[0,1]$ be given such that $\frac{\partial^{2} f}{\partial x^{2}}, \frac{\partial^{2} f}{\partial y^{2}}$ and $\frac{\partial^{4} f}{\partial x^{2} \partial y^{2}}$ are bounded on $] 0,1[\times] 0,1[$. The following composite Bernstein cubature formula

$$
\begin{aligned}
\int_{0}^{1} \int_{0}^{1} f(x, y) d x d y= & \frac{1}{m n(p+1)(q+1)} \sum_{k=1}^{m} \sum_{j=1}^{n} \sum_{h=0 l=0}^{p} \sum_{l=0}^{q} f\left(\frac{k p-p+h}{m p}, \frac{j q-q+l}{n q}\right) \\
& +R_{m, n}[f] .
\end{aligned}
$$

holds, were $R_{m, n}[f]$ satisfies (1.10).

Using (1.11) it is possible to approximate $\int_{0}^{1} \int_{0}^{1} f(x, y) d x d y$ which the desired precision $\varepsilon$, imposing the condition $\left|R_{m, n}[f]\right|<\varepsilon$.

The focus of the present paper is to extend the results from [4] in order to obtain a composite Bernstein type cubature formula on any bidimensional interval $[a, b] \times$ $[c, d]$. Finally, numerical examples and comparisons with other known cubature formulas will be provided. 


\section{MAIN RESULTS}

Let $a, b, c, d \in \mathbb{R}$ be given such that $a<b, c<d$ and $\mathbb{N}$ be the set of positive integers.

Lemma 1. The Bernstein bivariate polynomial associated to $f \in C[a, b] \times[c, d]$ is expressed for any $(x, y) \in[a, b] \times[c, d]$ and any $m, n \in \mathbb{N}$ by

$$
\bar{B}_{m, n} f(x, y)=\sum_{k=0}^{m} \sum_{j=0}^{n} \bar{p}_{m, k}(x) \bar{p}_{n, j}(y) f\left(a+k \cdot \frac{b-a}{m}, c+j \cdot \frac{d-c}{n}\right)
$$

where

$$
\begin{aligned}
& \bar{p}_{m, k}(x)=\frac{1}{(b-a)^{m}}\left(\begin{array}{l}
m \\
k
\end{array}\right)(x-a)^{k}(b-x)^{m-k}, \\
& \bar{p}_{n, j}(y)=\frac{1}{(d-c)^{n}}\left(\begin{array}{l}
n \\
j
\end{array}\right)(y-c)^{j}(d-y)^{n-j}
\end{aligned}
$$

are the Bernstein fundamental polynomials.

Proof. The vectorial function $(t, s) \rightarrow\left(\frac{x-a}{b-a}, \frac{y-c}{d-c}\right)$ transform the bidimensional interval $[a, b] \times[c, d]$ into $[0,1] \times[0,1]$. Taking the above and definitions $(1.1),(1.2)$ into account one arrives to (2.1), (2.2).

For any $f \in C[a, b] \times[c, d], m, n \in \mathbb{N}$ the equality

$$
f(x, y)=\bar{B}_{m, n} f(x, y)+\bar{R}_{m, n} f(x, y)
$$

is the Bernstein approximation formula on $[a, b] \times[c, d]$. Applying Theorem 1.3, for the remainder term of (2.3) follows

Lemma 2. If $f \in C^{2,2}[a, b] \times[c, d]$ such that $\frac{\partial^{2} f}{\partial x^{2}}, \frac{\partial^{2} f}{\partial y^{2}}, \frac{\partial^{4} f}{\partial x^{2} \partial y^{2}}$ are bounded on $] a, b[\times] c, d[$, the following

$$
\begin{aligned}
\left|R_{m, n} f(x, y)\right| & \leq \frac{(x-a)(b-x)}{2 m(b-a)^{2}} M_{1}[f] \\
& +\frac{(y-c)(d-y)}{2 n(d-c)^{2}} M_{2}[f] \\
& +\frac{(x-a)(b-x)(y-c)(d-y)}{4 m n(b-a)^{2}(d-c)^{2}} M_{3}[f]
\end{aligned}
$$

holds, for any $(x, y) \in[a, b] \times[c, d]$, where 


$$
\begin{aligned}
& M_{1}[f]=\sup _{(x, y) \in I \times J}\left|\frac{\partial^{2} f}{\partial x^{2}}(x, y)\right|, \\
& M_{2}[f]=\sup _{(x, y) \in I \times J}\left|\frac{\partial^{2} f}{\partial y^{2}}(x, y)\right|, \\
& M_{3}[f]=\sup _{(x, y) \in I \times J}\left|\frac{\partial^{4} f}{\partial x^{2} \partial y^{2}}(x, y)\right|
\end{aligned}
$$

and $I \times J=[a, b] \times[c, d]$.

Consider now the bidimensional interval $[a, b] \times[c, d]$ divided in $m \cdot n$ equally spaced subintervals

$$
\begin{gathered}
I_{k} \times J_{j}=\left[a+(k-1) \cdot \frac{b-a}{m}, a+k \cdot \frac{b-a}{m}\right] \times\left[c+(j-1) \cdot \frac{d-c}{n}, c+j \cdot \frac{d-c}{n}\right], \\
k=\overline{1, m}, j=\overline{1, n} .
\end{gathered}
$$

In each such type of interval one considers the distinct knots $\left(x_{h}, y_{l}\right)$, $h=\overline{0, p}, l=\overline{0, q}$, where

$$
x_{h}=a+(k p-p+h) \cdot \frac{b-a}{m p}, y_{l}=c+(j q-q+l) \cdot \frac{d-c}{n q}, h=\overline{0, p}, l=\overline{0, q} .
$$

Applying Lemma 2.1 on $I_{k} \times J_{j}$, follows the following bivariate Bernstein type polynomial

$$
\begin{gathered}
\bar{B}_{p, k, q, j} ; f(x, y)=\frac{m^{p} \cdot n^{q}}{(b-a)^{p}(d-c)^{q}} \sum_{k=0}^{p} \sum_{l=0}^{q}\left(\begin{array}{l}
p \\
k
\end{array}\right)\left(\begin{array}{l}
q \\
l
\end{array}\right) \\
\left(x-a-(k-1) \cdot \frac{b-a}{m}\right)^{k}\left(a+k \cdot \frac{b-a}{m}-x\right)^{p-k}\left(y-c-(j-1) \cdot \frac{d-c}{n}\right)^{l}\left(a+j \cdot \frac{d-c}{n}-y\right)^{q-l} \\
f\left(a+(k p-p+h) \cdot \frac{b-a}{m p}, c+(j q-q+l) \cdot \frac{d-c}{n q}\right)
\end{gathered}
$$

On any bidimensional interval

$$
I_{k} \times J_{j}=\left[a+(k-1) \cdot \frac{b-a}{m}, a+k \cdot \frac{b-a}{m}\right] \times\left[c+(j-1) \cdot \frac{d-c}{n}, c+j \cdot \frac{d-c}{n}\right]
$$

holds the bivariate Bernstein approximation formula

$$
f(x, y)=\bar{B}_{p, k, q, j} f(x, y)+\bar{R}_{p, k, q, j} f(x, y) .
$$

Applying Lemma 2.2 we get the following upper bound estimation for the remainder term of (2.8)

$$
\left|\bar{R}_{p, k, q, j} f(x, y)\right|
$$




$$
\begin{aligned}
& \leq \frac{m\left(x-a-(k-1) \cdot \frac{b-a}{m}\right)\left(a+k \cdot \frac{b-a}{m}-x\right)}{2(b-a)^{2}} M_{1}^{\prime \prime}[f]+ \\
& +\frac{n\left(y-c-(j-1) \cdot \frac{d-c}{n}\right)\left(c+j \cdot \frac{d-c}{n}-y\right)}{2(d-c)^{2}} M_{2}^{\prime \prime}[f]+ \\
& +\frac{m n\left(x-a-(k-1) \cdot \frac{b-a}{m}\right)\left(a+k \cdot \frac{b-a}{m}-x\right)\left(y-c-(j-1) \cdot \frac{d-c}{n}\right)\left(c+j \cdot \frac{d-c}{n}-y\right)}{4(b-a)^{2}(d-c)^{2}}
\end{aligned}
$$

where

$$
\begin{aligned}
& M_{1}^{\prime \prime}[f]=\sup _{(x, y) \in I_{k} \times J_{j}}\left|\frac{\partial^{2} f}{\partial x^{2}}(x, y)\right|, \\
& M_{2}^{\prime \prime}[f]=\sup _{(x, y) \in I_{k} \times J_{j}}\left|\frac{\partial^{2} f}{\partial y^{2}}(x, y)\right|, \\
& M_{3}^{\prime \prime}[f]=\sup _{(x, y) \in I_{k} \times J_{j}}\left|\frac{\partial^{4} f}{\partial x^{2} \partial y^{2}}(x, y)\right| .
\end{aligned}
$$

Theorem 6. Let $I_{k} \times J_{j}=\left[a+(k-1) \cdot \frac{b-a}{m}, a+k \cdot \frac{b-a}{m}\right] \times\left[c+(j-1) \cdot \frac{d-c}{n}, c+j \cdot \frac{d-c}{n}\right]$ and $x_{k}, y_{l}$ given at (2.6). The coefficients of the Bernstein type cubature formula

$$
\iint_{I_{k} \times J_{j}} f(x, y) d x d y=\sum_{h=0}^{p} \sum_{l=0}^{q} \bar{A}_{h, k, l, j} f\left(x_{k}, y_{l}\right)+\bar{R}_{k j}[f]
$$

are expressed by

$$
\bar{A}_{h, k, l, j}=\frac{(b-a)(d-c)}{m n(p+1)(q+1)} .
$$

Proof. Integrating (2.8) on $I_{k} \times J_{j}$ and taking (2.7) into account, yields

$$
\begin{gathered}
\bar{A}_{h, k, l, j}=\frac{m^{p} \cdot n^{q}}{(b-a)^{p}(d-c)^{q}}\left(\begin{array}{l}
p \\
k
\end{array}\right)\left(\begin{array}{l}
q \\
l
\end{array}\right) \iint_{I_{k} \times J_{j}}\left(x-a-(k-1) \cdot \frac{b-a}{m}\right)^{k}\left(a+k \cdot \frac{b-a}{m}-x\right)^{p-k} \\
\cdot\left(y-c-(j-1) \cdot \frac{d-c}{n}\right)^{l}\left(c+j+\frac{d-c}{n}-y\right)^{q-l} d x d y .
\end{gathered}
$$

Denoting

$$
A_{p}=\frac{m^{p}}{(b-a)^{p}}\left(\begin{array}{l}
p \\
h
\end{array}\right) \int_{I_{k}}\left(x-a-(k-1) \cdot \frac{b-a}{m}\right)^{k}\left(a+k \cdot \frac{b-a}{m}-x\right)^{p-k} d x
$$


respectively

$$
A_{q}=\frac{n^{q}}{(d-c)^{q}}\left(\begin{array}{l}
q \\
l
\end{array}\right) \int_{J_{j}}\left(y-c-(j-1) \cdot \frac{d-c}{n}\right)^{l}\left(c+j+\frac{d-c}{n}-y\right)^{q-l} d y
$$

we have

$$
\bar{A}_{h, k, l, j}=A_{p} \cdot A_{q} .
$$

To computing $A_{p}$ one makes the change of variable $x=a+(k-1) \frac{b-a}{m}+t \frac{b-a}{m}$, which leads to

$$
\begin{aligned}
A_{p} & =\frac{m^{p}}{(b-a)^{p}}\left(\begin{array}{l}
p \\
h
\end{array}\right) \frac{(b-a)^{p}}{m^{p}} \cdot \frac{b-a}{m} \int_{0}^{1} t^{h}(1-t)^{p-h} d t \\
& =\left(\begin{array}{l}
p \\
h
\end{array}\right) \frac{b-a}{m} B(h+1, p-h+1)
\end{aligned}
$$

were $B(h+1, p-h+1)$ is the Euler function of first kind. Taking its well known properties into account, one arrives to

$$
A_{p}=\left(\begin{array}{l}
p \\
h
\end{array}\right) \frac{b-a}{m} \cdot \frac{h !(p-h) !}{(p+1) !}=\frac{b-a}{m(p+1)} .
$$

In a similar way one obtains $A_{q}=\frac{d-c}{n(q+1)}$ and then, taking the equality $\bar{A}_{h, k, l, j}=$ $A_{p} \cdot A_{q}$ into account follows (2.12).

Theorem 7. Let $f \in C^{2,2}[a, b] \times[c, d]$ such that exist $\frac{\partial^{4} f}{\partial x^{2} \partial y^{2}}$ on $I_{k} \times J_{j}$ and $\frac{\partial^{2} f}{\partial x^{2}}, \frac{\partial^{2} f}{\partial y^{2}}, \frac{\partial^{4} f}{\partial x^{2} \partial y^{2}}$ are bounded on $I_{k} \times J_{j}, k=\overline{1, m}, j=\overline{1, n}$. Then, the following upper bound estimation for the remainder term of (2.11)

$$
\begin{gathered}
\left|\bar{R}_{k . j}[f]\right| \leq \frac{(b-a)(d-c)}{12 m^{2} n} M_{1}^{\prime \prime}[f]+\frac{(b-a)(d-c)}{12 m n^{2}} M_{2}^{\prime \prime}[f]+ \\
+\frac{(b-a)(d-c)}{144 m^{2} n^{2}} M_{3}^{\prime \prime}[f]
\end{gathered}
$$

holds, where $M_{1}^{\prime \prime}[f], M_{2}^{\prime \prime}[f], M_{3}^{\prime \prime}[f]$ are defined at (2.10).

Proof. The inequality (2.13) follows integrating the approximation formula (2.8) and taking the inequalities (2.9) into account.

The main result of the paper is the following 
Theorem 8. Let $f \in C^{2,2}[a, b] \times\left[c, d\left[\right.\right.$ be given such that exist $\frac{\partial^{4} f}{\partial x^{2} \partial y^{2}}$ on ]$a, b[\times] c, d\left[\right.$ and $\frac{\partial^{2} f}{\partial x^{2}}, \frac{\partial^{2} f}{\partial y^{2}}, \frac{\partial^{4} f}{\partial x^{2} \partial y^{2}}$ are bounded on $] a, b[\times] c, d[$. Then, the following composite Bernstein type cubature formula

$$
\int_{a}^{b} \int_{c}^{d} f(x, y) d x d y=\frac{(b-a)(d-c)}{m n(p+1)(q+1)} \sum_{k=i}^{m} \sum_{j=i}^{n} \sum_{h=0}^{p} \sum_{l=0}^{q} f\left(x_{h}, y_{l}\right)+R_{m, n}[f]
$$

holds, where $x_{h}, y_{l}$ are defined at (2.6) while the remainder term verifies the inequality (1.10).

Proof. Adding the Bernstein type cubature formulas (2.11) for $k=\overline{1, m}$, $j=\overline{1, n}$ one arrives to $(2.14)$.

\section{Remark 1.}

(i) For $a=0, b=1$ one refinds the results from [7].

(ii) It is immediate that $\lim _{m, n \rightarrow \infty}\left|R_{m, n}[f]\right|=0$, which proves that

$$
\begin{gathered}
\lim _{\substack{m \rightarrow \infty \\
n \rightarrow \infty}} \frac{(b-a)(d-c)}{m n(p+1)(q+1)} \\
\sum_{k=i}^{m} \sum_{j=i}^{n} \sum_{h=0}^{p} \sum_{l=0}^{q} f\left(a+(k p-p+h) \cdot \frac{b-a}{m p}, c+(j q-q+l) \cdot \frac{d-c}{n q}\right)= \\
=\int_{a}^{b} \int_{c}^{d} f(x, y) d x d y .
\end{gathered}
$$

\section{NUMERICAL EXAMPLES}

In [9] the authors introduce cubature formulas for two-variables function with boundary-layer components to evaluate the integral

$$
I_{f}=\int_{a}^{b} \int_{c}^{d} f(x, y) d x d y
$$

For a uniform grid on the domain $[a, b] \times[c, d]$ with nodes $\left(x_{i}, y_{j}\right), i=0,1, \ldots, m$, $j=0,1, \ldots, n$, with steps $h_{1}$ and $h_{2}$ in $x$ and $y$, respectively, let $u_{i, j}=f\left(x_{i}, y_{j}\right)$.

Let us denote:

Trapezoidal Rule (TR)

$T R_{f}=\frac{h_{1} h_{2}}{4} \sum_{i, j}\left(u_{i+1, j+1}+u_{i, j+1}+u_{i, j}+u_{i+1, j}\right), 0 \leq i<m, 0 \leq j<n$ 
Simpson's Rule (SR)

$$
\begin{gathered}
S R_{f}=\frac{h_{1} h_{2}}{9} \sum_{i, j}\left(16 u_{i, j}+4 u_{i+1, j}+4 u_{i-1, j}+4 u_{i, j+1}+4 u_{i, j-1}+4 u_{i+1, j+1}\right. \\
\left.+u_{i-1, j-1}+u_{i+1, j-1}+u_{i-1, j+1}\right), \quad i=1,3, \cdots, m-1, \quad j=1,3, \cdots, n-1
\end{gathered}
$$

Bernstein Rule (BR)

$$
\begin{gathered}
B R_{f}=\frac{(b-a)(d-c)}{m n(p+1)(q+1)} \\
\sum_{k=i}^{m} \sum_{j=i}^{n} \sum_{h=0}^{p} \sum_{l=0}^{q} f\left(a+(k p-p+h) \cdot \frac{b-a}{m p}, c+(j q-q+l) \cdot \frac{d-c}{n q}\right)
\end{gathered}
$$

To demonstrate the accuracy of our new numerical cubature formula, we compare the Bernstein Rule (BR) with Trapezoidal Rule (TR) and Simpson's Rule (SR) by using the following test functions:

$$
\begin{gathered}
f_{1}:[0,2] \times[0,2] \rightarrow \mathbb{R}, f_{1}(x, y)=e^{-\left(x^{2}+y^{2}\right)} \\
f_{2}:[0,5] \times[0,3] \rightarrow \mathbb{R}, f_{2}(x, y)=e^{-2(x+y)} \sin (4 x+4 y) \\
f_{\varepsilon}[0,1] \times[0,1] \rightarrow \mathbb{R}, f_{\varepsilon}(x, y)=\left(1-e^{\frac{-x}{\varepsilon}}\right)\left(1-e^{\frac{-2 y}{\varepsilon}}\right)(1-x)(1-y)+ \\
+\cos \left(\frac{\pi x}{2}\right) e^{-y}, \varepsilon \in(, 1] .
\end{gathered}
$$

The last function $f_{\varepsilon}$ is studied in [9] for the values of $\varepsilon=1, \varepsilon=10^{-1}, \varepsilon=10^{-2}$, $\varepsilon=10^{-3}, \varepsilon=10^{-4}$ and $\varepsilon=10^{-5}$, by using Trapezoidal Rule (TR), Simpson's Rule (SR) and the new methods introduced by the author.

Let us denote the values of the integrals by

$$
\begin{gathered}
I_{f_{1}}=\int_{0}^{2} \int_{0}^{2} f_{1}(x, y) d x d y, \quad I_{f_{2}}=\int_{0}^{5} \int_{0}^{3} f_{2}(x, y) d x d y, \\
I_{f_{\varepsilon}}=\int_{0}^{1} \int_{0}^{1} f_{\varepsilon}(x, y) d x d y
\end{gathered}
$$

and the errors by

$$
e T R_{f}=\left|I_{f}-T R_{f}\right|, \quad e S R_{f}=\left|I_{f}-S R_{f}\right|, e B R_{f}=\left|I_{f}-B R_{f}\right| .
$$

The numerical results obtained for $m=64, n=64, p=5$ and $q=5$ are presented in Table 1 and Table 2. In these tables, $e-m$ means $10^{-m}$.

The Mathcad 14.0 package was used to generate these numerical results. 
Table 1. Errors for $f_{1}$ and $f_{2}$

\begin{tabular}{cccc}
\hline & $\mathrm{eTR}_{f}$ & $\mathrm{eSR}_{f}$ & $\mathrm{eBR}_{f}$ \\
\hline$f_{1}$ & $1.05 \mathrm{e}-5$ & $6.84 \mathrm{e}-9$ & $2.10 \mathrm{e}-6$ \\
$f_{2}$ & $1.40 \mathrm{e}-5$ & $1.89 \mathrm{e}-6$ & $1.47 \mathrm{e}-6$ \\
\hline
\end{tabular}

Table 2. Errors for $f_{\varepsilon}$

\begin{tabular}{cccc}
\hline$\varepsilon$ & $e T R_{f_{\varepsilon}}$ & $e S R_{f_{\varepsilon}}$ & $e B R_{f_{\varepsilon}}$ \\
\hline 1 & $2.68 \mathrm{e}-5$ & $6.65 \mathrm{e}-11$ & $5.37 \mathrm{e}-6$ \\
$10^{-1}$ & $2.87 \mathrm{e}-4$ & $1.42 \mathrm{e}-6$ & $5.76 \mathrm{e}-5$ \\
$10^{-2}$ & $2.71 \mathrm{e}-3$ & $6.95 \mathrm{e}-4$ & $5.54 \mathrm{e}-4$ \\
$10^{-3}$ & $7.01 \mathrm{e}-3$ & $4.43 \mathrm{e}-3$ & $1.91 \mathrm{e}-3$ \\
$10^{-4}$ & $7.76 \mathrm{e}-3$ & $5.11 \mathrm{e}-3$ & $2.59 \mathrm{e}-3$ \\
$10^{-5}$ & $7.76 \mathrm{e}-3$ & $5.18 \mathrm{e}-3$ & $2.59 \mathrm{e}-3$ \\
\hline
\end{tabular}

\section{CONCLUSions}

In Table 1 and Table 2 one can see that Bernstein cubature formula revised is better than trapezoidal cubature formula for all functions studied. Even if the Simpson's cubature formula is of order $(2,2)$ and Bernstein cubature formula is of order $(1,1)$, the results obtained by using our revised formula are better for the cases of functions $f_{2}$ and $f_{\varepsilon}$, with $\varepsilon=10^{-2}, 10^{-3}, 10^{-4}, 10^{-5}$.

\section{REFERENCES}

[1] D. Bărbosu, "The approximation of multivariate functions by using boolean sums of linear positive operators of interpolatory type," in The approximation of multivariate functions by using boolean sums of linear positive operators of interpolatory type. Cluj-Napoca: Risoprint, 2002.

[2] D. Bărbosu, "On the Schurer-Stancu approximation formula." Carpathian J. Math, vol. 21, 2005.

[3] D. Bărbosu and D. Miclăuş, "On the composite Bernstein type cubature formula." Gen. Math., vol. 18, pp. 73-81, 2010.

[4] D. Bărbosu and D. Miclăuş, "On the composite Bernstein-type quadrature formula." Rev. Anal. Numer. Theor Approx., vol. 39, pp. 3-7, 2010.

[5] D. Bărbosu and O. T. Pop, "On the Bernstein bivariate approximation formula." Carpathian J. Math, vol. 24, pp. 293-298, 2008.

[6] D. Bărbosu and O. T. Pop, “A note on the Bernstein's cubature formula." Gen. Math., vol. 17, pp. 161-172, 2009.

[7] D. D. Stancu, "Quadrature formulas constructed by using certain linear operators." in Numerical Integration. Birkhăuser, 1982, pp. 241-251.

[8] D. D. Stancu, G. Coman, and P. Blaga, Numerical Analysis and Approximation Theory. Presa Univ. Clujeană, Cluj-Napoca(Romanian: II, 2002.

[9] A. I. Zadorin, "Cubature Formulas for a Two-Variable Function with Boundary-Layer Components." Computational Mathematics and Mathematical Physics, vol. 53, pp. 1808-1818, 2013. 
Authors' addresses

\section{Dan Bărbosu}

Department of Mathematics and Computer Science, North University Center at Baia Mare, Technical University of Cluj-Napoca, Victoriei 76, 430122 Baia Mare, Romania

E-mail address: barbosudaneyahoo.com

\section{Gheorghe Ardelean}

Department of Mathematics and Computer Science, North University Center at Baia Mare,Technical University of Cluj-Napoca, Victoriei 76, 430122 Baia Mare, Romania

E-mail address: ardelean_g@cunbm.utcluj.ro 